\title{
A WILDLIFE MONITORING SYSTEM BASED ON TIANDITU AND BEIDOU: IN CASE OF THE TIBETAN ANTELOPE
}

\author{
Zhang Hongping ${ }^{\mathrm{a},}$ *, Huang Wei ${ }^{\mathrm{a}}$, Wei Dong ${ }^{\mathrm{b}}$, Jiang Jie ${ }^{\mathrm{b}}$ \\ aNational Geomatics Center of China, 28 Lianhuachi West Road, Haidian District, Beijing, China, 100830 \\ bIntegrated Geographic Information Center of Shanxi Province, 136 Yingze Street, Taiyuan. China 03001
}

\author{
Commission IV, WG IVI/5
}

KEY WORDS: Tianditu, Beidou, GPS, Wildlife Monitoring, Realtime

\begin{abstract}
:
Positioning and tracking wildlife is already being an effective way to collect biological information for research and species of wildlife protection. The common technologies of tracking wildlife are divided into several categories, such as radio tracking technology, GPS tracking system, radio frequency identification technology (RFID), and SIM card based technology. Some positive results achieved from these technologies, but there are some problems in location accuracy, price of the system. Taking the case of the protection of the Tibetan antelope, this paper introduces a wildlife monitoring system based on Tianditu and Beidou navigation satellite system. The system consists of two parts: real-time location system and 3D display system. The practical application demonstrates that the system is stable, and data transmission is reliable with lower construction cost, which can improve the capability of national rare wildlife monitoring and protection effectively.
\end{abstract}

\section{INTRODUCTION}

The Tibetan antelope, as the national first-grade attentive protected species, is mainly distributed over the Qinghai-Tibet Plateau (Tibet, Qinghai, Gansu and Xinjiang), and is also listed in the U.N. Convention on International Trade in Endangered Species of Wild Fauna and Flora. For a long time, the Tibetan antelope is acknowledged as the typical indication species to Qinghai-Tibet plateau natural ecosystem, and has become the important object of research among scholars. Tibetan antelopes are of great value in scientific research, ecological balance, even humane and and aesthetic aspects. Therefore, there is a need for tracking and protecting the Tibetan antelope. Nowadays, radio tracking technology, GPS tracking technology, RFID technology, SIM card based tracking technology are the main technologies in the field of wildlife research and protection on international. The Argos tracking system was firstly applied to researching migration rules of Tibetan antelopes in Shaanxi Institute of Zoology from 2007 to 2010, which is built as a satellite communications system by the Centre National d' Etudes Spatiales (CNES) of France, NASA and NOAA for data collection and location tracking. But there are many disadvantages such as low frequency and precision of positioning, inconvenience of data acquisition and high cost. In order to restore the routes of migration and giving birth of the Tibetan antelope, a wildlife monitoring system must be developed including global, all-weather, continuous real-time and precision positioning, data acquisition and processing, 3D-Map display and analysis and other 
functions, so as to provide scientific basis for the future monitoring and protection of the Tibetan antelope.

TIANDITU is the website providing "one-stop" geospatial information services to personal users, enterprises, professional agencies and governments via public networks such as Internet, mobile communication network, etc. It is the public version of the National Platform for Common GeoSpatial Information Services, which is created by National Administration of Surveying, Mapping and Geoinformation of China (NASG) as an important part of the geospatial framework for digital China aims at promoting the geographic information resources sharing, and improving the ability and efficiency for services.

BeiDou Navigation Satellite System is China's global navigation satellite system which has been developed independently. It is not only equipped global, all-weather, continuous real-time and precision navigation and positioning capabilities, but also has good antijamming and security performance

Therefore, a wildlife monitoring system for monitoring and protecting Tibetan antelopes based on Tianditu and Beidou was built. The system is not only achieve real-time monitoring without any blind spot for all day, but also can display the migration routes on the $3 \mathrm{D}$ digital map.

\section{SYSTEM OUTLINE DESIGN}

\subsection{System Overall Structure}

As shown in Figure 1, the system mainly realized the collecting realtime positioning data from the Tibetan antelopes and integrate them into Tianditu 3D display system to show the entire migration, including two parts: Beidou real-time location system for the Tibetan antelope and Tianditu 3D display system.

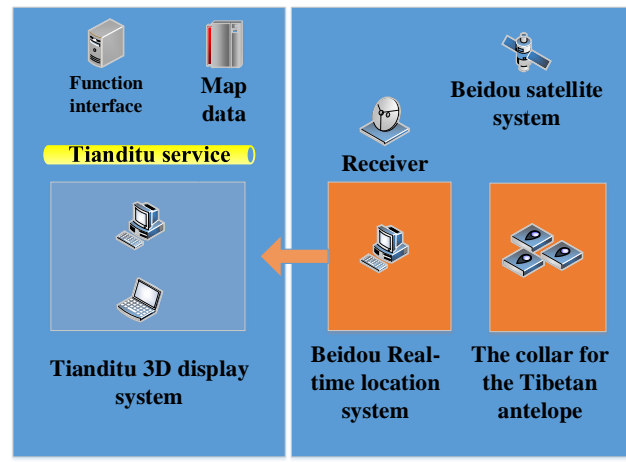

Figure 1: System overall structure
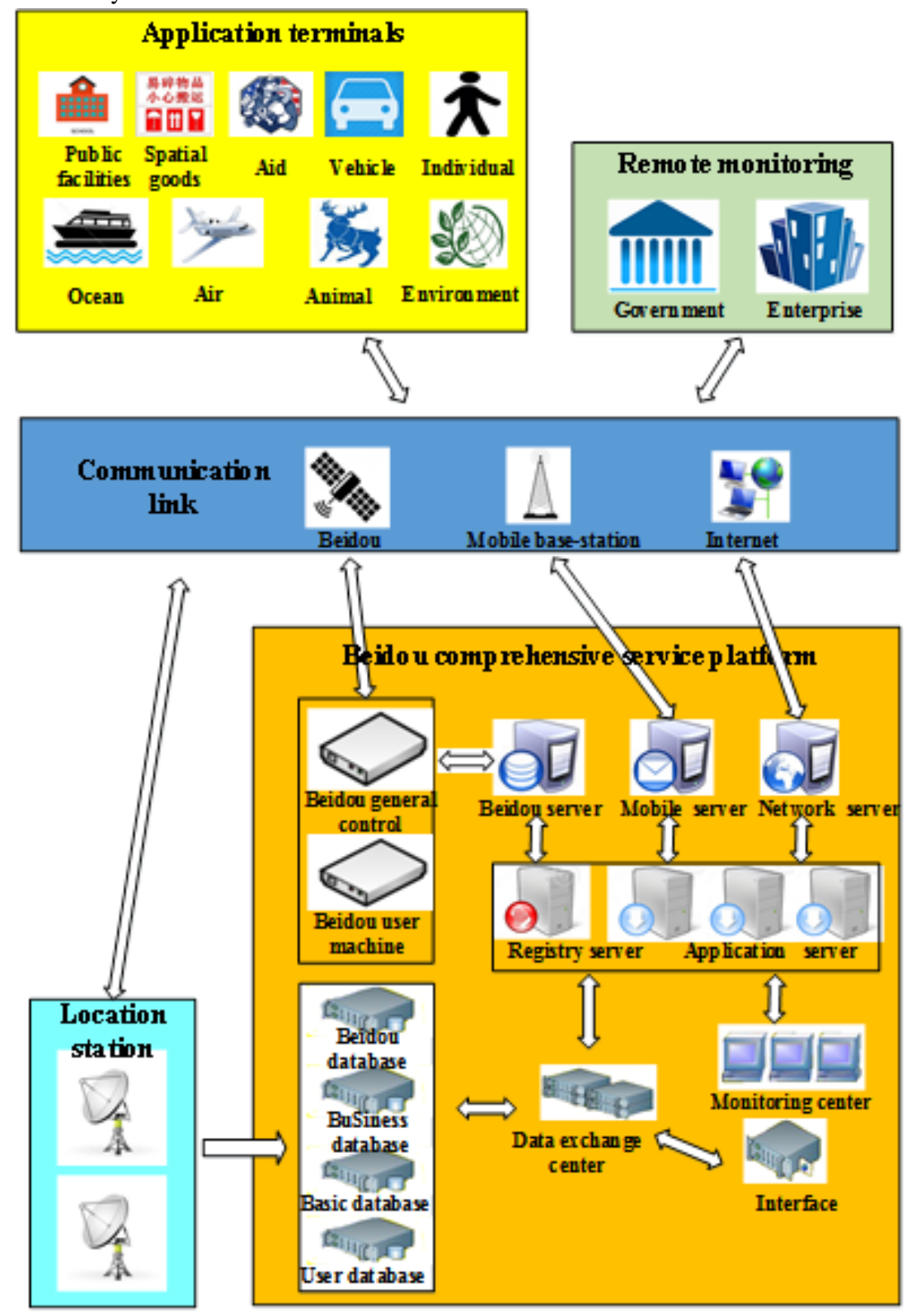

Figure 2: BeiDou Navigation Satellite System frame diagram 
2.2 Beidou Real-time Location System For The

\section{Tibetan Antelope}

Beidou real-time location system for the Tibetan antelope is composed of two parts: locating terminals (Beidou collar) and monitoring platform. The locating terminals achieve the functions of location, data acquisition, data distribution, and the monitoring platform, and the monitoring platform is responsible to receive, record, save the data from the locating terminals.

Through the low power chip solution with the advantages of fast capture, location, communication and high-efficiency power management, it can reduce the power dissipation, shorten running time, and control standby current, and support up to 18 months to work.

As shown in Figure 2, the monitoring platform, composed of Beidou general control, servers, and databases, which is responsible for real-time monitoring and receiving location information from information is saved in standard form in real time, and Tianditu 3D display system can obtain and draw the routes in time.

\subsection{Tianditu Three-dimensional Display System}

Tianditu three-dimensional display system is an important carrier of Tibetan Antelope positioning, tracking and visualization. The system is composed ofthe following modules: a) Map service: realization the map service of images and three dimensional terrain loading, supports both online and offline; b) BeiDou location data loading: analyzing and loading the location data from the Beidou real-time location system which receives coordinates of the Tibetan antelopes. c) Three dimensional browsing: providing functions such as roam, rotation, location with coordinates, and custom flight; d) Terrain analysis: including height, distance and area measurement; e) Analysis of Tibetan antelopes: providing analysis functions of migration path for different populations of Tibetan antelope and distribution of different species of antelope in different periods. The monitoring result of Tibetan antelope is shown in figure 3.

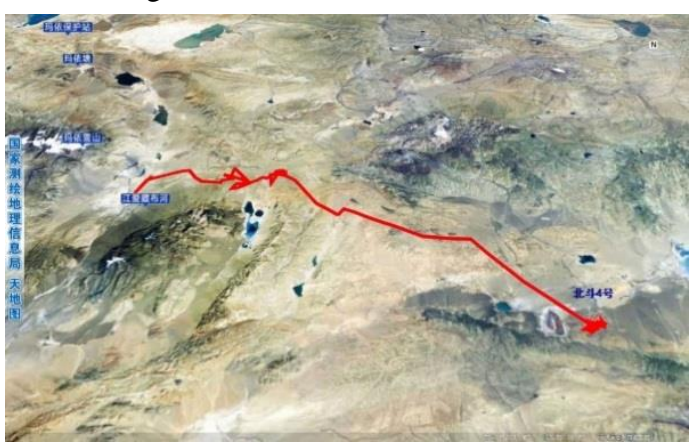

Figure 3: Migratory path of Tibetan antelopes

\section{KEY TECHNOLOGIES}

\subsection{Long-term Monitoring of BeiDou Navigation Satellite System in Real-time}

Tibetan antelopes inhabit Tibet Plateau where there is no public network and location information can't be sent back to the monitor platform easily, although almost accurate location can be found by GPS and BeiDou passive positioning technique. Problems in monitoring antelope remain unsolved. BeiDou RDSS system can not only provide relatively accurate location information, but also send it back to the monitor platform in real-time through BeiDou satellites.

However, since using bi-direction transmission for Beidou RDSS positioning and communication, the standby time is very short (Common Beidou RDSS Terminals standby time is up to 7 days), and the migratory cycle of Tibetan antelopes is about one year.

Thus, according to the application scenarios, the functions of BeiDou terminals are simplified to guarantee 18 months' working time. Using the function of location report to tracking Tibetan antelopes, which reduces working current and time of Beidou modules. In addition, the time and frequency will be pre-set based on the movement patterns of Tibetan antelopes, and the terminal will be in standby state after finishing a given task to make maximum save of energy consumption. 


\subsection{Three Dimensional Display and Analysis}

Although Tianditu supports BeiDou satellites signals importing, map view and virtual roaming, functions still needed to be expanded to meet the system's demands. Due to the characteristics of the point location data and high frequency, extra functions such as data filter and inquiry are added to draw the routes automatically with the location data at a time of the choosing, which can demonstrate the whole migration trajectory of the Tibetan antelopes with the same ID.

The perspective management of three dimensional flight is provided, so that users can edit every view while flying in the scene, and fly along with the migration route, even simulate their migration state. Also, configuration files of the perspectives can be imported or output so as to be connected to TV, and this increases reusability and inheritance of the view.

\section{CONCLUSION}

Beidou Navigation Satellite System, Tibetan Antelope tracking system and Tianditu platform introduced in this paper are all created by China, which effectively improve China's ability to track and protect rare animals.

On the technical side of things, the system makes full use of Beidou bilateral communication technology and has solved the problem to get wildlife's location in real time. Attempts have been made to prolong working time and duration of Beidou terminals in a bad environment. BeiDou navigation satellite system with fully independent intellectual property, high performance and reliability can successfully replace France's Argos in the field of wildlife protection, which has absolute advantages in location accuracy, real-time transmission and data security.

Tianditu three dimensional display system has provided comprehensive services including data processing, visualization, analysis and custom flight, and it can support researchers to monitor and protect Tibetan antelopes efficiently.
The system has been applied in the special program of Tibetan antelopes in CCTV (China Central TeleVision). As Beidou and Tianditu technology develops, the integrated application of them in the field of wildlife protection will be wider and deeper, which will make more contributions to protect the ecological environment of China.

\section{REFERENCE}

WU Xiao min, ZHANG Hong feng, 2011. Resources Regarding Populations of Tibetan Antelope (Pantholops hodgsoni) and the Status of Its Protection. Chinese Journal of Nature 33(3), 143-148+154+188189.

Horback, K. M., Miller, L. J., Andrews, J., Kuczaj, S. A., \& Anderson, M. (2012). The effects of GPS collars on African elephant (Loxodonta africana) behavior at the San Diego Zoo Safari Park. Applied Animal Behaviour Science 142(1), 76-81.

Zhang xianfeng, Wang Ding, \&Yang Jian,etc(1996). Study on Radio-Tracking Finless porpoise Neophocaena Phocaenoides, At the Yangtze River. Acta Ecologica Sinica 16(5), pp, 490-496.

Dyo, V., Ellwood, S. A., Macdonald, D. W., Markham, A., Mascolo, C., Pásztor, B., ... \& Yousef, K. (2010, November). Evolution and sustainability of a wildlife monitoring sensor network. In Proceedings of the 8th ACM Conference on Embedded Networked Sensor Systems (pp. 127-140). ACM.

Dyo, V., Ellwood, S. A., Macdonald, D. W., Markham, A., Trigoni, N., Wohlers, R., ... \& Yousef, K. (2012). WILDSENSING: Design and deployment of a sustainable sensor network for wildlife monitoring. ACM Transactions on Sensor Networks (TOSN), 8(4), 29. 